\title{
Placenta Percreta Presenting as an Acute Obstetric Emergency in Mid-second Trimester: A Case Report
}

\author{
Ashima Kesri ${ }^{1}$, Neelima Choudhary ${ }^{2}$, Jagadish C Sharma ${ }^{3}$
}

\begin{abstract}
Aim: To know the risks and management of placenta percreta in patients with previous lower segment cesarean section (LSCS) with postabortal hemorrhage.

Background: Placenta percreta, a form of adherent placenta, is associated with significant postabortal hemorrhage often requiring emergency hysterectomy. Majority of these cases are seen in patients with a history of previous LSCS with anterior low-lying placenta.

Case description: A 29-year-old female with previous two LSCS came at 19 weeks 4 days of gestational age with complaints of leaking per vaginum since 7 days and mild pain abdomen. Her ultrasonography showed single live intrauterine fetus (SLIUF) with anterior upper segment placenta with no evidence of accretion. She went into labor spontaneously and expelled a male baby weighing $300 \mathrm{gm}$. Subsequently, she had postabortal hemorrhage with the placenta in situ. Medical management of post-abortal hemorrhage (PAH) was given but she went into shock with massive blood loss. After stabilization of the patient with iv fluids and colloids, she was taken up for emergency laparotomy, in view of retained placenta with previous two LSCS with postabortal hemorrhage as medical management failed. On laparotomy, the placenta was anterior and was invading the wall of the uterus up to the serosa. As she was bleeding torrentially, an emergency hysterectomy was done without any delay in decision-making. She was transfused with four packed red blood cells (PRBC) and four fresh frozen plasma (FFPs). Her postoperative period was uneventful and she was discharged on day 7 in astable condition. Histopathological findings confirmed the absence of the placental basal plate and the presence of trophoblastic tissue in the myometrium and serosa.

Conclusion: Good anticipation and timely decision are very important in decreasing maternal morbidity and mortality in cases of PPH with adherent placentas, like in other obstetric emergencies.

Clinical significance: We wish to highlight the importance of good clinical knowledge with timely decision by OB/Gyn team in a patient with $\mathrm{PPH}$ and placenta percreta encountered in the mid-second trimester.

Keywords: Hysterectomy, Placenta accrete spectrum, Placenta percreta, Postabortal hemorrhage.

Journal of South Asian Federation of Obstetrics and Gynaecology (2021): 10.5005/jp-journals-10006-1881
\end{abstract}

\section{BACKGROUND}

Placenta percreta, a form of morbidly adherent placenta, occurs when the placenta invades into the myometrium and extends up to the uterine serosa. The incidence of placenta percreta is 1 in 21,000 pregnancies and a majority of these cases are seen in patients with a history of previous LSCS. We present a case of placenta percreta in a patient with previous two LSCS with normal ultrasonography.

\section{Case Description}

A 29-year-old female, G3P2L2 with 19 weeks 4 days of gestational age with previous two LSCS, was admitted in the labor room with complaints of leaking per vaginum since 7 days and mild pain abdomen since 1 day. She had no complaints of bleeding per vaginum. Her previous two LSCS were 10 and 9 years back. There was no history of previous abortions or suction and evacuations. She had no significant medical past history. On examination, her general condition was fair, BP 120/84 mm of mercury in the right arm in semirecumbent position, pulse rate $84 /$ minute regular, and palpable at all peripheral pulses. There was no pallor, icterus, cyanosis, clubbing, or edema. Per abdomen examination was suggestive of 20 weeks size uterus, irritable with no scar tenderness, fetal heart rate of $140 \mathrm{bpm}$ on fetal Doppler. Per speculum examination showed clear liquor draining through os. Per vaginum examination had os one finger tight with absent membranes. Her routine blood investigations were sent. Ultrasonography was suggestive of SLIUF
${ }^{1,3}$ Department of Obstetrics and Gynaecology, Employees State Insurance Corporation (ESIC) Medical College and Hospital, Faridabad, Haryana, India

${ }^{2}$ Department of Obstetrics and Gynaecology, Employees State Insurance Corporation (ESIC) Medical College and Hospital, Delhi, India Corresponding Author: Neelima Choudhary, Department of Obstetrics and Gynaecology, Employees State Insurance Corporation (ESIC) Medical College and Hospital, Delhi, India, Phone: +91 9868350813, e-mail: choudhary.neelima13@gmail.com

How to cite this article: Kesri A, Choudhary N, Sharma JC. Placenta Percreta Presenting as an Acute Obstetric Emergency in Midsecond Trimester: A Case Report. J South Asian Feder Obst Gynae 2021;13(2):135-137.

Source of support: Nil

Conflict of interest: None

18 weeks 4 days with absent liquor. The placenta was the anterior upper segment with no evidence of accreta on color Doppler ultrasonography. Poor prognosis of the fetus was explained, and informed consent was taken. She went into labor spontaneously and expelled a male baby weighing $300 \mathrm{gm}$ within 6 hours of admission. Subsequently, she had PAH with the placenta in situ. Controlled cord traction was applied to remove the placenta but the placenta 
could not be separated. Medical management of PPH was tried with injection oxytocin and prostadin but failed. Ten units of oxytocin was given in the cord for separation of placenta but failed. Within a short span of time of 20 minutes postdelivery, she went into shock with massive blood loss. Immediate resuscitation with intravenous fluids and other supportive management was done. An immediate decision was taken for emergency laparotomy with adequate blood products with informed consent. On laparotomy, placenta was anterior and was invading the wall of the uterus up to serosa with no evidence of bladder invasion. She was bleeding torrentially so an emergency hysterectomy was done. Blood loss was about 2.5 litres. She was transfused with four PRBC and four FFPs. The patient was shifted to ICU. Her postoperative period was uneventful and she was discharged on day 7 in stable condition. Histopathological findings confirmed the absence of the placental basal plate and the presence of trophoblastic tissue in the myometrium and serosa.

\section{Discussion}

Placenta accrete spectrum, is a complication of pregnancy, where the placenta abnormally attaches to the wall of the uterus with partial or complete invasion in form of placenta increta, placenta percreta, and placenta accreta (Fig. 1). This is a life-threatening obstetrical emergency with increased incidence from 0.12 to $0.31 \%$ in the last few years and the mortality rate of nearly $7.0 \% .{ }^{1}$ It is associated with increased maternal morbidity which includes multiple blood transfusions, urinary tract injury, hysterectomy, ICU admission, sepsis, and prolonged hospital stay. ${ }^{2}$

There are multiple risk factors associated with the placenta accrete spectrum, with the most common being increasing rate of cesarean sections in the past few years. ${ }^{3-5}$ A systematic review by Marshall et al. showed the rate of placenta accrete spectrum increases from $0.3 \%$ in women with one previous cesarean delivery to $6.74 \%$ in women with five or more cesarean deliveries. ${ }^{6}$ In addition to previous cesarean delivery, uterine curettage or hysteroscopy surgery, myomectomy, and endometrial ablation may result in further localized decidual defect and consequently abnormal placentation. Another most important risk factor is placenta previa and the risk of placenta accrete in women with placenta previa is $3,11,40,61$, and $67 \%$, for the first, second, third, fourth, and fifth or more cesarean, respectively. ${ }^{7}$

The diagnosis of placenta accrete spectrum should be made during the antenatal period in order to have better outcomes. The primary diagnostic modality is antenatal ultrasonography.
Although features of accrete may be present as early as the first trimester on ultrasonography; however, most women are diagnosed in the second and third trimesters. Ideally, women with risk factors for placenta accrete spectrum should be screened carefully by obstetrician-gynecologist and confirmed diagnosis should be made by color Doppler ultrasonography.

The accuracy of sonography using greyscale and color Doppler techniques for antenatal diagnoses of placenta accrete varies widely in different studies with a sensitivity ranging between 33 and $100 \%$. A systematic review by D'Antonio F, including 23 studies and 3,707 pregnancies, noted an average sensitivity of 90.72\% (95\% Cl, 87.2-93.6) and specificity of $96.94 \%(95 \% \mathrm{Cl}$, 96.3-97.5\%). ${ }^{8}$ Another study from the past shows that sonography has a positive predictive value of $78 \%$ and a negative predictive value of $94 \%$ but some authors suggested that sonography might detect only around 33\% of cases of placenta accrete/ increta. ${ }^{9,10}$ Overall, ultrasound has a good diagnostic accuracy in identifying the spectrum of placenta accrete with sensitivities of $90.6,93.0,89.5$, and $81.2 \%$ for placenta accreta, increta, accreta/ increta, and percreta, respectively; the corresponding specificities were 97.1, 98.4, 94.7, and 98.9\%. Placental lacunae that appear as intraplacental sonolucent spaces with disruption of the interface between the bladder wall and uterine serosa (bladder line) are the most reliable diagnostic sonographic findings. Color flow Doppler demonstrating turbulent ("chaotic") flow and/or bridging vessels are valuable confirmatory findings.

Diagnosis is confirmed by histopathology. Postabortal histological findings show placental villi anchored directly on, or invading into or through, the myometrium, without an intervening decidual plate.

Management of placenta accrete spectrum involves a standardized approach with a multidisciplinary team well trained for the management of placenta accrete spectrum. ${ }^{11,12}$ Timing of decisions is very important in order to prevent any maternal risks. A good and experienced obstetrician, obstetric anesthetist, urologist, critical care expert, and a full-time operational blood bank are the basic prerequisites for the management of the placenta accrete spectrum. There are various treatment options available for the management of placenta accrete depending on the expertise of each center, like hysterectomy, leave the placenta in situ followed by methotrexate or perform local resection. Based on multiple studies, surgical intervention has been suggested as the first line of treatment of placenta percreta, as hysterectomy is mostly required in $93 \%$ of cases.

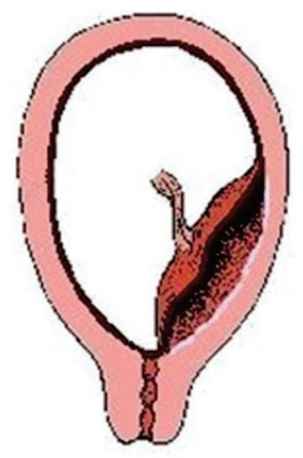

Normal

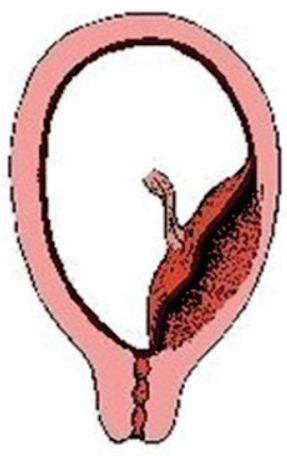

Accreta

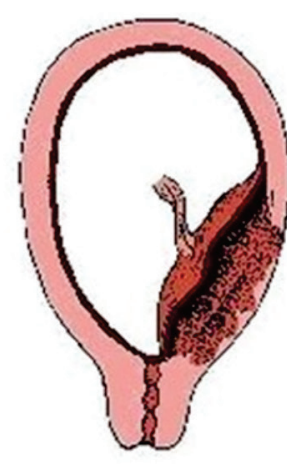

Increta

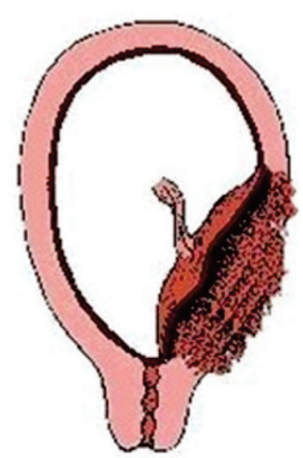

Percreta

Fig. 1: Abnormalities of attachment of the placenta. 


\section{Conclusion}

Placenta percreta is potentially a life-threatening condition for a mother. It usually occurs at the site of the previous section scar. Proper antenatal screening with good ultrasonography can avoid major complications. The decision to perform hysterectomy or conservative management is to be individualized depending upon the clinical condition of patient and expertise of gynecologist. Timely decision is most important in morbidly adherent placenta as in any other obstetric emergencies. We also emphasize on keeping the placenta accrete spectrum in the differential diagnosis of retained placenta for a timely management of such catastrophe and improved maternal outcome, specially even when all previous imaging has ruled out placenta accrete spectrum, as no imaging modality has $100 \%$ sensitivity.

\section{Clinical Significance}

We wish to highlight the importance of good clinical knowledge with a timely decision by OB/Gyn team in a patient with PPH and placenta percreta encountered in the mid-second trimester.

\section{Acknowledgments}

We would like to thank the Department of OB/GYN, ESIC Medical College and Hospital, Faridabad, for their valuable support and cooperation.

\section{References}

1. Zhang $D$, Siqin $Y$, Yanyan $H$, et al. Risk factors, outcome and management survey of placenta accreta disorders in 153 cases: $A$ five-year experience from a hospital of Shanghai, China. Int J Clin Exp Med 2017;10(8):12509-12516.

2. Oyelese $Y$, Smulian JC. Placenta previa, PAS disorders, and vasa previa. Obstet Gynecol 2006;107(4):927-941. DOI: 10.1097/01. AOG.0000207559.15715.98.
3. Usta IM, Hobeika EM, Musa AA, et al. Placenta previa-accreta: risk factors and complications. Am J Obstet Gynecol 2005;193:1045-1049. DOI: 10.1016/j.ajog.2005.06.037.

4. Eshkoli T, Weintraub AY, Sergienko R, et al. Placenta accreta: risk factors, perinatal outcomes, and consequences for subsequent births. Am J Obstet Gynecol 2013;208(3):219.e1-219.e7. DOI: 10.1016/j. ajog.2012.12.037.

5. Bowman ZS, Eller AG, Bardsley TR, et al. Risk factors for placenta accreta: a large prospective cohort. Am J Perinatol 2014;31(9):799804. DOI: 10.1055/s-0033-1361833.

6. Marshall NE, Fu R, Guise JM. Impact of multiple cesarean deliveries on maternal morbidity: a systematic review. Am J Obstet Gynecol 2011;205(3):262.e1-262.e8. DOI: 10.1016/j.ajog.2011.06.035.

7. Silver RM, Landon MB, Rouse DJ, et al. Maternal morbidity associated with multiple repeat cesarean deliveries. National Institute of Child Health and Human Development Maternal-Fetal Medicine Units Network. Obstet Gynecol 2006;107(6):1226-1232. DOI: 10.1097/01. AOG.0000219750.79480.84.

8. D'Antonio F, lacovella C, Bhide A. Prenatal identification of invasive placentation using ultrasound: systematic review and meta-analysis. Ultrasound Obstet Gynecol 2013;42(5):509-517. DOI: 10.1002/ uog.13194.

9. Finberg HJ, Williams JW. Placenta accreta: prospective sonographic diagnosis in patients with placenta previa and prior cesarean section. J Ultrasound Med 1992;11(7):333-343. DOI: 10.7863/ jum.1992.11.7.333.

10. Lam G, Kuller J, McMahon M. Use of magnetic resonance imaging and ultrasound in the antenatal diagnosis of placenta accreta. J Soc Gynecol Investig 2002;9(1):37-40. DOI: 10.1016/s10715576(01)00146-0.

11. Shamshirsaz AA, Fox KA, Salmanian B, et al. Maternal morbidity in patients with morbidly adherent placenta treated with and without a standardized multidisciplinary approach. Am J Obstet Gynecol 2015;212(2):218.e1-218.e9. DOI: 10.1016/j. ajog.2014.08.019.

12. Silver RM, Barbour KD. Placenta accreta spectrum: accreta, increta, and percreta. Obstet Gynecol Clin North Am 2015;42(2):381-402. DOI: 10.1016/j.ogc.2015.01.014. 